\title{
QUALIDADE DA ALFACE CRESPA MINIMAMENTE PROCESSADA ACONDICIONADA EM ATMOSFERA MODIFICADA
}

\author{
Quality of minimally processed crisp head lettuce packaged under modified atmosphere
}

\author{
Rosana Mendes Roversi ${ }^{1}$, Maria Lucia Masson ${ }^{2}$
}

\begin{abstract}
RESUMO
A alface Crespa (Lactuca sativa L.) foi minimamente processada, tendo suas folhas destacadas e higienizadas, embalada em sacos plásticos de polietileno com modificação ativa da atmosfera dentro do pacote. Duas atmosferas foram aplicadas, variando-se o nível de $\mathrm{CO}_{2}$ em 10 e $15 \%$, reduzindo nos dois casos o $\mathrm{O}_{2}$ a $5 \%$. Após o acondicionamento, a alface foi imediatamente refrigerada. Comparou-se a qualidade da alface com e sem atmosfera modificada durante o armazenamento refrigerado. Monitoraram-se a variação da concentração de $\mathrm{O}_{2}$ e $\mathrm{CO}_{2}$ no interior das embalagens e a vida-útil da alface pela análise sensorial e microbiológica e a variação de sólidos solúveis. A influência da atmosfera modificada no crescimento de microrganismos e na variação de sólidos solúveis não foi relevante. Entretanto, essa tecnologia reduziu as taxas de respiração e, conseqüentemente, minimizou as perdas de qualidade sensorial da alface durante todo o armazenamento. A vida-útil da alface em atmosfera modificada foi estendida em até cinco dias além daquela alcançada pela alface acondicionada em atmosfera normal.
\end{abstract}

Termos para indexação: Alface, processamento mínimo, conservação, atmosfera modificada.

\begin{abstract}
The crisp head lettuce (Lactuca sativa L.) was minimally processed, with the leaves being detached and cleaned, packaged in plastic bags made of polyethylene with modified atmosphere inside the bag. Two different atmospheres were applied: 10 and $15 \%$ of $\mathrm{CO}_{2}$, with the $\mathrm{O}_{2}$ level being reduced in both cases to $5 \%$. After the packing, the lettuce was immediately refrigerated. The product quality with and without modified atmosphere was compared during the storage time. It was accompanied the changes in $\mathrm{O}_{2}$ and $\mathrm{CO}_{2}$ concentration inside the packaging and the product shelf life, through sensorial and microbiological analysis, and soluble solids variation. The influence of modified atmosphere in microorganisms grown and in soluble solids variation was not relevant. However this technology reduced the lettuce respiration rates and the quality sensorial loss during storage. The shelf life of lettuce in modified atmosphere was extended in five days in comparison with the ones packaged in normal atmosphere.
\end{abstract}

Index terms: Lettuce, minimally process, conservation, modified atmosphere.

(Recebido para publicação em 23 de outubro de 2003 e aprovado em 13 de janeiro de 2004)

\section{INTRODUÇÃO}

Os vegetais minimamente processados são produtos similares aos frescos, que passam por operações tais como limpeza, classificação, pelagem e corte, as quais podem ser realizadas antes do branqueamento em uma linha de processamento convencional. Como não sofrem qualquer tipo de tratamento térmico, são constituídos por tecidos vivos, que continuam apresentando o mecanismo da respiração (ROLLE e CHISM, 1987).

O metabolismo dos vegetais minimamente processados é mais intenso do que nos tecidos intactos dos vegetais "in natura", devido ao processamento para redução em pedaços. Nesse caso, a taxa de respiração aumenta consideravelmente; além disso, a maior exposição do conteúdo celular devida aos cortes promove aumento da suscetibilidade à oxidação enzimática e à contaminação microbiológica. A velocidade da respiração está diretamente relacionada com a senescência do vegetal, pois nesse processo as substâncias estruturais das células vegetais são catabolizadas, resultando em $\mathrm{CO}_{2}$, água e energia. A redução da velocidade de respiração é obtida principalmente por meio da refrigeração. Portanto, a refrigeração é essencial na conservação dos vegetais minimamente processados, sendo essa categoria referida por Wiley (1997) como RMP - refrigerados minimamente processados.

A redução da taxa de respiração vegetal pode se otimizada por modificação da atmosfera ao redor da alface, quer seja por redução do oxigênio ou aumento do gás carbônico $\mathrm{O}$ princípio de ação da redução do teor de oxigênio e aumento do gás carbônico, aliados a baixas

\footnotetext{
1. Engenheira de Alimentos/UNICAMP, mestre em Tecnologia de Alimentos - UFPR - Rua Adelaide Stresser Scheleder, 135 , casa 12 - Jardim Social - Curitiba, PR

2. Engenheira Química/UFPR, doutora em Engenharia de Alimentos - UNICAMP, professora adjunto do Departamento de Tecnologia Química da UFPR.
} 
temperaturas, fundamenta-se na redução de elementos imprescindíveis do processo de respiração, provocando a redução das velocidades das reações biológicas envolvidas no metabolismo, que, por sua vez, são responsáveis pelo envelhecimento dos tecidos celulares (BRECHT, 1980; KADER, 1986; SHEWFELT, 1986; ZAGORY, 1988; WILEY, 1997). A atmosfera modificada apresenta ação anti-enzimática e pode promover a inibição da polifenoloxidase, enzima responsável pelo escurecimento de compostos fenólicos devido à elevada concentração de $\mathrm{CO}_{2}$ (MATEOS et al., 1993; HAMZA, et al. 1996).

O sucesso da Atmosfera Modificada (AM) consiste em manter o mais próximo ao ótimo os níveis reduzidos de $\mathrm{O}_{2}$ e elevados de $\mathrm{CO}_{2}$, para obter os efeitos benéficos de redução da taxa de respiração, sem promover efeitos negativos, como as desordens fisiológicas provocadas pelo excesso de gás carbônico (escurecimento e degradação dos tecidos,) ou ainda a substituição da respiração aeróbica pelo metabolismo anaeróbio, quando o teor de oxigênio é insuficiente para que a respiração ocorra. Além de promover a alteração do sabor e aroma por causa de seus produtos metabólitos, a anaerobiose acelera a senescência natural dos tecidos vegetais, promovendo rápida degradação do vegetal (SHEWFELT, 1986; KADER et al., 1989; ROLLE e CHISM, 1987; SOUZA et al., 2001).

A modificação da atmosfera pode ser obtida pela substituição do ar por uma composição gasosa previamente estabelecida, efetuando-se primeiramente o vácuo na embalagem. Esse tipo de modificação é denominado modificação ativa (BALLANTYNE et al., 1988).

A estabilização da atmosfera no interior da embalagem dependerá da respiração e da permeabilidade do $\mathrm{O}_{2}$ e $\mathrm{CO}_{2}$ através do filme da embalagem. Para tanto, o material de embalagem deverá, além das funções básicas de proteção, fracionamento e segurança, apresentar características de permeabilidade compatíveis com o metabolismo do produto a ser embalado, ou seja, promover a entrada de oxigênio que compense o seu consumo e permitir a saída do gás carbônico gerado pela respiração (ZAGORY e KADER, 1988; KADER et al., 1989). Entre os diversos materiais de embalagem existentes para acondicionamento de hortaliças, o PEBD (polietileno de baixa densidade) tem sido amplamente aplicado em razão de características de alta resistência à degradação química e da relativa alta permeabilidade aos gases, além de resposta favorável à termosselagem, disponibilidade e baixo custo (BALLANTYNE et al., 1988; KADER et al., 1989; SARANTÓPOULOS, 1996).

As respostas ao aumento de $\mathrm{CO}_{2}$ e diminuição de $\mathrm{O}_{2}$ são diferentes até entre variedades de uma mesma espécie, dependendo das características intrínsecas de cada vegetal e da difusividade dos gases nos tecidos (MATEOS, 1993; HEIMDAL, 1995; HAMZA, 1996; et al., LÓPEZ-GALVEZ, 1997). Segundo Lipton (1977) e Brecht (1980), a alface Crespa apresentou manchas escuras nas folhas quando foi submetida a níveis de $\mathrm{CO}_{2}$ maiores que $1 \%$, ao passo que a alface-romana pode tolerar até $12 \%$. Mais recentemente, Hamza et al. (1996), trabalhando com alface Romana minimamente processada, não detectou distúrbios fisiológicos nos tratamentos com concentrações de $1 \%$ a $3 \%$ de $\mathrm{O}_{2}$, e $5-10 \%$ de $\mathrm{CO}_{2}$.Por essa razão, é necessário particularizar o estudo para cada espécie, variedade ou tipo de vegetal de interesse do ponto de vista comercial.

A degradação dos carboidratos devida à respiração e à conversão de amido em açúcar pode ser afetada pela AM, o que promoveria alterações na variação dos sólidos solúveis durante o armazenamento. Além disso, essas alterações poderão refletir na característica sensorial dos produtos, indicando os níveis de metabolismo mantidos durante o armazenamento (YI WANG, 1990; ZAGORY e KADER, 1989; YAMASHITA, 1995).

A análise sensorial tem sido utilizada para determinação dos parâmetros de qualidade de alface, como sabor, aroma, murchamento (textura) e aparência, tendo como ferramenta escalas numéricas subjetivas. Na avaliação da aparência, os defeitos que exercem maior influência no julgamento são aqueles de origem enzimática, como o escurecimento marrom-ferrugem das nervuras e tecidos brancos e manchas marrons nas folhas e nas extremidades, cortados ou amassados (KADER et al., 1973; SHEWFELT, 1987; HEIMDAL, 1995).

Com o presente trabalho objetivou-se avaliar a influência da atmosfera modificada na redução da taxa de respiração da alface (Lactuca sativa L.) mediante avaliação da qualidade sensorial, variação de sólidos solúveis e crescimento microbiano, durante o armazenamento, comparando a vida-útil em relação ao produto sem atmosfera modificada. Além disso, objetivou-se monitorar a estabilidade da atmosfera ativamente modificada no acondicionamento da alface em sacos de PEBD pela determinação das concentrações de $\mathrm{O}_{2}$ e $\mathrm{CO}_{2}$ no interior das embalagens. 


\section{MATERIAL E MÉTODOS}

\section{Matéria-prima}

A alface Crespa é uma hortaliça constituída por folhas bem consistentes, enrugadas e bastante repicadas nas bordas, não formando cabeça (exceto as crespas do tipo americanas) e pertence à espécie Lactuca sativa $L$. (FILGUEIRA, 2000).

A cultivar Verônica, com plantas crespas de grande porte, produzida na região metropolitana de Curitiba, foi adquirida diretamente do produtor, sem classificação quanto à categoria (defeitos e sujidade), sendo colhida pela manhã (5 horas) e transportada em caixas de madeira com cerca de 20 unidades cada uma, permanecendo cerca de quatro horas em temperatura ambiente, tempo esse que correspondeu ao período entre a colheita e o início do processamento.

\section{Embalagem}

Os sacos plásticos produzidos com filme PEBD (dimensões: $29 \times 25 \mathrm{~cm}$ ) foram adquiridos nesse formato com os três lados soldados pelo fabricantes. As características do filme estão descritas na Tabela 1. Para espessura, utilizou-se um micrômetro de ponta Starret, realizando-se 25 repetições para obtenção da média. As taxas de $\mathrm{TPO}_{2}$ foram determinadas por método colorimétrico, segundo procedimento descrito na norma ASTM D 3985-95 e as taxas de $\mathrm{TPCO}_{2}$ foram determinadas por método de aumento da concentração.

\section{Atmosfera modificada}

As composições gasosas 1 e 2 foram cedidas pela AGA - fabricante de gases especiais, em cilindros de $1,5 \mathrm{Kg}$ (Tabela 2).

A mistura gasosa (ATM3: $21 \%$ de $\mathrm{O}_{2}$ e $0,03 \%$ de $\mathrm{CO}_{2}$ ) foi aplicada para simular a composição do oxigênio e gás carbônico presentes no ar e foi obtida com a perfuração manual dos sacos com agulhas de $\varnothing 0,5 \mathrm{~mm}$ a cada $3 \mathrm{~cm}$ (16 furos a cada $0,81 \mathrm{~m}^{2}$ ), os quais foram denominados de "alface controle". Garantiu-se que, com essa perfuração, o ar saísse e entrasse com facilidade através da embalagem sob leve pressão manual.

\section{Processamento}

Inicialmente a alface recebeu uma pré-lavagem para retirada de resíduos da terra em água corrente. A seguir, as folhas foram separadas manualmente da raiz com faca de aço inoxidável, de forma que permanecessem inteiras, eliminando-se as folhas rasgadas, amassadas, escurecidas nas extremidades ou com necroses.

TABELA 1 - Espessura e taxa de permeabilidade ao $\mathrm{O}_{2}\left(\mathrm{TPO}_{2}\right)$ e ao $\mathrm{CO}_{2}\left(\mathrm{TPCO}_{2}\right)$ a $23^{\circ} \mathrm{C}$, a seco e 1 atm de gradiente de pressão parcial de gás permeante.

\begin{tabular}{lccc}
\hline \multicolumn{1}{c}{ Características } & Média & $\mathbf{I V}^{\mathbf{4}}$ & $\mathbf{C V}^{\mathbf{5}}$ \\
\hline $\left.\mathrm{TPO}_{2} \mathrm{~cm}^{3}(\mathrm{CNTP}) / \mathrm{m}^{2} / 24 \mathrm{~h}\right)^{1}$ & 2.844 & $2.747-2.942$ & 5 \\
$\left.\mathrm{TPCO}_{2} \mathrm{~cm}^{3}(\mathrm{CNTP}) / \mathrm{m}^{2} / 24 \mathrm{~h}\right)^{2}$ & 12.073 & $10.633-13.640$ & 11 \\
Espessura $(\mu \mathrm{m})^{3}$ & 60 & $55-64$ & 5 \\
\hline
\end{tabular}

${ }^{1} \mathrm{TPO}_{2}$ : resultados médios de duas determinações

${ }^{2}$ TPCO: resultados médios de três determinações;

${ }^{3}$ Média referente a vinte e cinco determinações

${ }^{4}$ IV - Intervalo de variância

${ }^{5} \mathrm{CV}$ : coeficiente de variação 
TABELA 2 - Misturas gasosas aplicadas nos testes.

\begin{tabular}{cc}
\hline Código & Alface \\
\hline ATM1 & $5 \% \mathrm{O}_{2} \times 10 \% \mathrm{CO}_{2} \times 85 \% \mathrm{~N}_{2}$ \\
ATM2 & $5 \% \mathrm{O}_{2} \times 15 \% \mathrm{CO}_{2} \times 80 \% \mathrm{~N}_{2}$ \\
ATM3(Controle) & $21 \% \mathrm{O}_{2} \times 0,03 \% \mathrm{CO}_{2} \times 78,96 \% \mathrm{~N}_{2}$ \\
\hline
\end{tabular}

$\mathrm{Na}$ sequiência, as folhas foram mergulhadas em água clorada com hipoclorito de sódio a $150 \mathrm{mg} . \mathrm{L}^{-1}$, preparada a partir de hipoclorito de sódio comercial a $12 \%$ e mantidas em imersão por 10 minutos para, então, serem enxaguadas em água potável, centrifugadas em centrifuga de cesto TREU (modelo 666.103B) a 850 rpm, por 30 segundos, pesadas e acondicionadas nos sacos, com 150 gramas cada um. O teor de cloro ativo foi determinado pelo método descrito no Standard Methods for the Examination of Water and Waterwasher (APHA 2001).

A alteração da atmosfera foi realizada automaticamente no fechamento dos pacotes pela seladora a vácuo (marca Triporvac, modelo KV-999), que retira o ar do interior da embalagem mediante formação de vácuo na câmara e injeta a composição gasosa preparada. Após o fechamento, todas as amostras e a alface-controle (ATM3) selada sem vácuo e sem injeção de ar foram imediatamente armazenadas em refrigerador a $5-7^{\circ}$ e mantidos, assim, por 15 dias, período em que foram realizadas as avaliações.

\section{Determinação da atmosfera no interior das embalagens}

Foram tomadas medidas diárias da evolução dos teores de $\mathrm{O}_{2}$ e $\mathrm{CO}_{2}$ até o terceiro dia e, a partir de então, a cada dois dias, até que a alface apresentasse condições de consumo, utilizando-se o analisador de "headspace" marca ABISS, modelo VAK 12 MAP03. As médias foram obtidas da leitura de dois pacotes.

\section{Análise sensorial}

A avaliação da qualidade sensorial foi feita por equipe de oito julgadores previamente selecionados e treinados na descrição das características da alface e dos defeitos. O método utilizado foi Análise Descritiva Qualitativa - ADQ, por meio de escala hedônica de nove pontos para a aparência e defeitos (escurecimento da nervura central róseo-ferrrugem, manchas escuras pontuais e escurecimento das extremidades das folhas) e escala de cinco pontos para sabor, textura e aroma (KADER et al., 1973; MEILGAARD et al., 1991). Definiu-se que o limite de aceitação da alface para consumo no atributo aparência geral seria cinco, e para os demais atributos, dois. Os dados obtidos foram submetidos à análise de variância (ANOVA) e a comparação das médias, pelo teste de Tukey.

\section{Análise microbiológica}

A cada cinco dias, amostras foram retiradas de dois sacos e homogeneizadas para contagem total de bactérias totais (ágar padrão PCA) e contagem de fungos (dicloram rosa e bengala clorafenicol), segundo método oficial do APHA (2001).

\section{Determinação de sólidos solúveis (SS)}

Uma amostra de $50 \mathrm{~g}$ foi retirada de dois pacotes previamente homogeneizados, triturada em homogeneizador doméstico e filtrada em tecido de nylon para extração de suco. No suco, foram feitas as determinações de acordo com os métodos descritos no AOAC (1998) utilizando-se um refratômetro de bancada.

\section{Delineamento Experimental}

Utilizou-se o delineamento inteiramente casualizado em dois blocos (repetições), sendo os resultados sensoriais e de \%SS analisados pela ANOVA e comparados em cada período, a 5\% de significância.

\section{RESULTADOS E DISCUSSÃO}

A variação da composição gasosa no interior das embalagens pode ser observada nas Figuras 1 e 2, para $\mathrm{O}_{2}$ e $\mathrm{CO}_{2}$, respectivamente. Pode-se observar que a velocidade da respiração foi maior nos dois primeiros dias em conseqüência do acelerado consumo de $\mathrm{O}_{2}$ nesse período, o que pode ser devido ao gradiente de temperatura entre o refrigerador e a alface no inicio do armazenamento. Segundo Wiley (1997), a velocidade de respiração está diretamente relacionada com a temperatura, a qual, no experimento, foi sendo reduzida lentamente, visto que o processo foi em refrigerador doméstico sem circulação de ar e a troca térmica nessa situação ocorre por condução. 


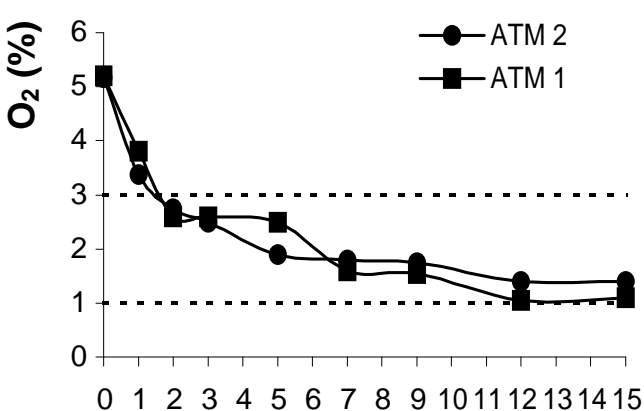

Tempo (dias)

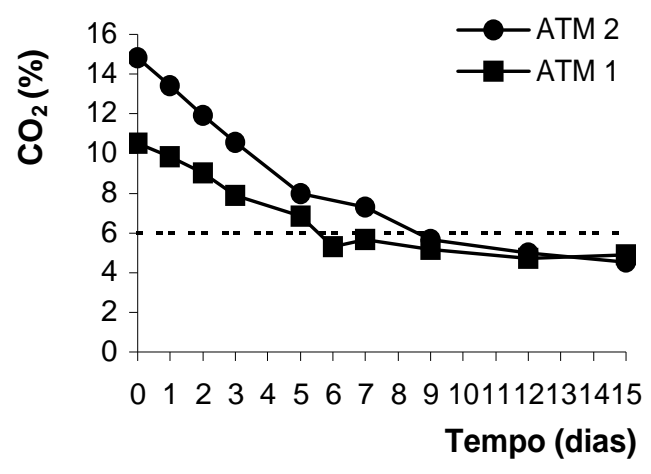

FIGURA 2 - Variação da concentração do gás carbônico.
Após esse período, houve uma maior estabilidade do gás e o nível de $\mathrm{O}_{2}$ permaneceu entre 1 e $3 \%$ até quinze dias de armazenamento, tanto nos produtos acondicionados em ATM1 quanto em ATM2 . Nos dois tratamentos, houve perda de $\mathrm{CO}_{2}$ através do filme; porém, a maior concentração inicial desse gás auxiliou sua manutenção em níveis maiores na alface com ATM2, que permaneceu até 9 dias acima da concentração mantida na alface em ATM1.

No atributo aparência, observa-se na Figura 3 a superioridade da alface acondicionada em ATM2, seguido pelo ATM1 e, por último, pelo ATM3 (ar), sendo suas médias estatisticamente diferentes $(\mathrm{p}<0,05)$ a partir do quinto dia de avaliação.

Comparando-se a avaliação de defeitos, pode-se observar uma relação direta entre a aparência e o escurecimento das folhas e da nervura (Figuras 3, 4 e 5). Segundo Heimdal (1995) e Kader et al. (1973), o escurecimento ferrugem-marrom das nervuras e o escurecimento pontual e das extremidades das folhas da alface estão relacionados com a atividade enzimática da polifenoloxidases. A minimização desses defeitos observados nas Figuras 4 e 5 pode estar associada à respectiva redução da atividade enzimática durante o armazenamento em ATM 1 e 2.

Comparando-se as Figuras 2 e 4, observa-se que, no mesmo período, com a concentraçao de $\mathrm{CO}_{2}$ na alface em ATM1 reduzida a 7\%, foi detectada na avaliação sensorial a incidência leve de escurecimento das nervuras. A intensidade dos defeitos aumentou à medida que o $\mathrm{CO}_{2}$ foi sendo reduzido nas embalagens. Segundo Mateos (1993) e Hamza et al. (1996), o efeito do incremento de $\mathrm{CO}_{2}$ está relacionado com a redução da atividade enzimática, em especial da polifenoloxidase. A ação anti-enzimática da atmosfera modificada, que resultou em menor incidência de escurecimento nas ner- vuras e nas folhas, foi mais eficiente, enquanto a concentração de $\mathrm{CO}_{2}$ manteve-se até $8 \%$.

A partir do décimo dia, a alface em ATM1 perde significativamente (5\% de significância) a qualidade sensorial no atributo aparência, sendo rejeitada pelo painel sensorial no décimo-quinto dia, ao passo que aquela mantida em ATM2 foi considerada aceitável por até quinze dias. A alface-controle foi rejeitada no atributo aparência no décimo dia de análise.

Não houve diferença significativa entre os demais atributos analisados nos produtos em ATM1 e ATM2, porém, ambos foram significativamente superiores ao produto em ATM3 em quase todos os atributos, exceto na avaliação de textura.

O efeito de inibição de fungos e bactérias na alface com atmosfera modificada não foi evidente. Observou-se que em até dez dias de estocagem não houve diferença significativa entre os valores obtidos (Tabelas 3 e 4), tanto para bactérias quanto para fungos.

No $15^{\circ}$ dia, houve uma redução de dois ciclos logarítmicos na carga microbiana (bactérias e fungos) no tratamento ATM2 em relação ao ATM1 e apenas um ciclo logaritmo no ATM3. Segundo Kader (1986), o efeito fungicida do $\mathrm{CO}_{2}$ depende da espécie de fungo existente na flora e da concentração do gás, e níveis abaixo de $1 \%$ ou acima de $10 \%$ promovem significante redução do crescimento de fungos. Esse resultado foi confirmado por Barriga (1991) em alface 'Iceberg' minimamente processada estocada sob atmosfera controlada $\left(3 \%\right.$ de $\mathrm{O}_{2}$ e $10 \%$ de $\mathrm{CO}_{2}$ ). Porém, Jacksen (1999), analisando diferentes vegetais MP, notificou que em baixas concentrações de $\mathrm{CO}_{2}(2-3 \%)$ a influência da $\mathrm{AM}$ na qualidade microbiológica não foi sempre óbvia, porém, notaram a extensão da fase lag no crescimento de microrganismos deteriorantes em alface 'Iceberg'. 


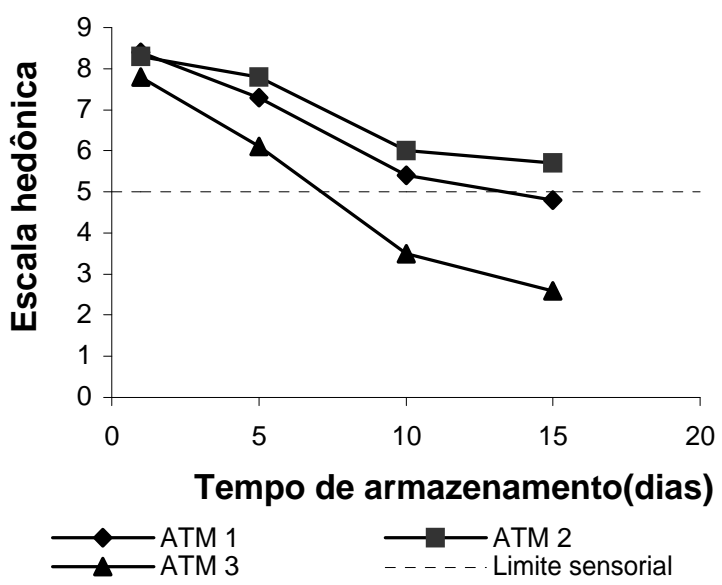

FIGURA 3 - Avaliação da aparência da alface durante o armazenamento.

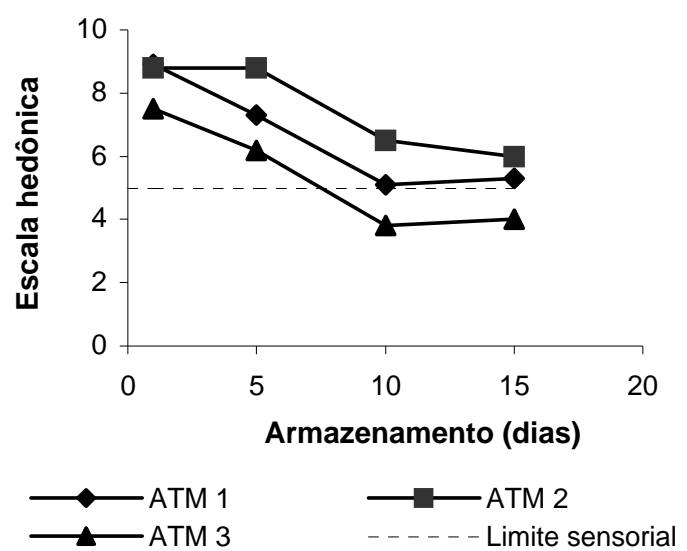

FIGURA 4 - Escurecimento das nervuras.

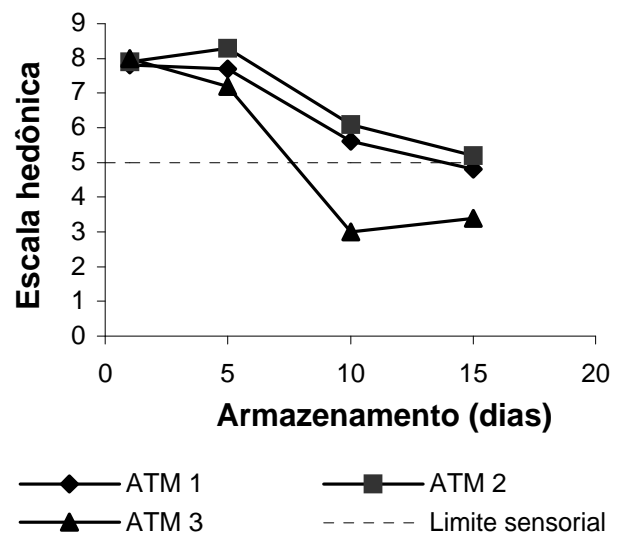

FIGURA 5 - Escurecimento das folhas (centro e extremidades).

TABELA 3 - Contagem total de bactérias em alface Crespa MP.

\begin{tabular}{cccc}
\hline \multirow{2}{*}{ Tempo } & \multicolumn{3}{c}{ Tratamentos (ufc/g $\mathbf{g}^{\mathbf{1}}$} \\
\cline { 2 - 4 } & ATM1 (1) & ATM2 (2) & ATM3 (ar) \\
\hline 0 & $1,12.10^{4}$ & $1,12.10^{4}$ & $1,12.10^{4}$ \\
5 & $8,77.10^{5}$ & $3,32.10^{5}$ & $9,60.10^{5}$ \\
10 & $1,55.10^{8}$ & $1,35.10^{8}$ & $5,10.10^{7}$ \\
15 & $2,10.10^{7}$ & $2,90.10^{6}$ & $2,50.10^{7}$ \\
\hline
\end{tabular}




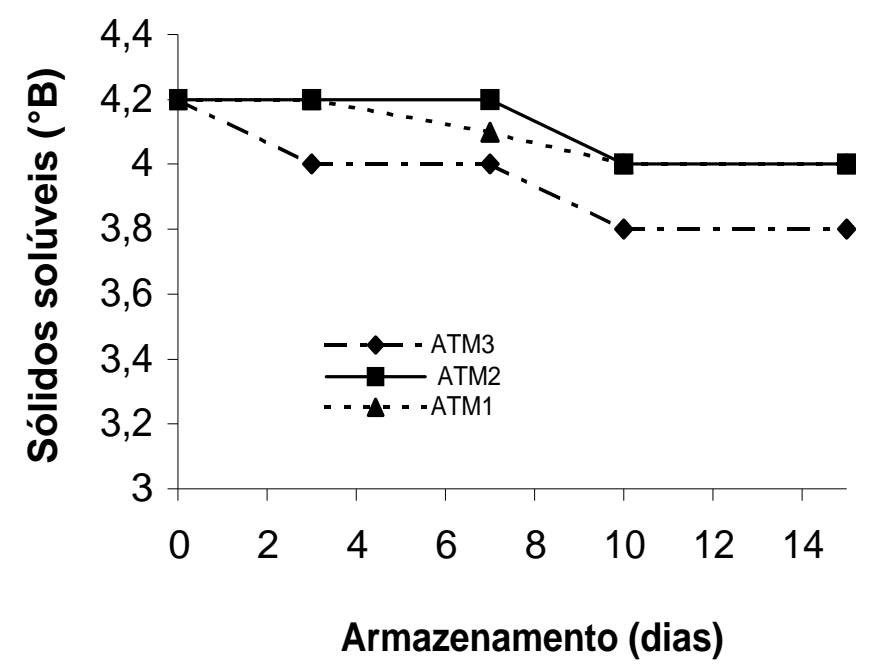

FIGURA 6 - Variação dos sólidos solúveis da alface armazenada.

TABELA 4 - Contagem total de fungos em alface Crespa MP.

\begin{tabular}{cccc}
\hline \multirow{2}{*}{ Tempo } & \multicolumn{3}{c}{ Tratamentos (ufc/g $\mathbf{1}$ ) } \\
\cline { 2 - 4 } & ATM1 (1) & ATM2 (2) & ATM3 (ar) \\
\hline 0 & $3,90.10^{3}$ & $3,90.10^{3}$ & $3,90.10^{3}$ \\
5 & $4,25.10^{5}$ & $1,65.10^{5}$ & $2,55.10^{4}$ \\
10 & $6,53.10^{7}$ & $1,42.10^{8}$ & $7,30.10^{7}$ \\
15 & $1,60.10^{6}$ & $4,10.10^{6}$ & $5,90.10^{6}$ \\
\hline
\end{tabular}

1ufc/g: unidade formadora de colônias por grama

\section{CONCLUSÕES}

Não houve estabilidade da AM nas embalagens de alface MP com a utilização do filme PEBD 60, principalmente quanto à concentração de $\mathrm{CO}_{2}$, devido ao desequilíbrio entre a respiração do produto e a taxa de transmissão de $\mathrm{O}_{2}$ e $\mathrm{CO}_{2}$ através do filme.

Apesar dessa deficiência, a alface acondicionada em AM apresentou qualidade sensorial superior durante todo o armazenamento em relação ao produto controle; no tratamento com ATM2, obtiveram-se quinze dias de vida-útil, ou seja, pelo menos cinco dias a mais do que a alface acondicionada sem atmosfera modificada. Não houve influência significativa da AM no desenvolvimento microbiano. Embora não estatisticamente significativa, houve interferência da AM no metabolismo respiratório da alface em razão da menor variação de sólidos solúveis observada durante o armazenamento.

\section{AGRADECIMENTOS}

Ao CETEA - ITAL, pela realização das analises de permeabilidade e espessura do filme; AGA - Termoindustrial, pelo empréstimo de equipamentos e fornecimento dos gases e CEPPA, pelas analises microbiológicas.

\section{REFERÊNCIAS BIBLIOGRÁFICAS}

ASSOCIATION OF OFFICIAL ANALYTICAL CHEMISTS. Bacteriological analytical manual. 8. ed. Gaithersburg, 1998.

APHA. Compendium of methods for the microbiological examination of foods. 4. ed. Washington: Frances Pounch Downes, 2001.

BALLANTYNE, A.; STARK, R.; SELMAN, J. D. Modified atmosphere packaging of shredded lettuce. International Journal of Food Science and Technology, Oxford, v. 23, p. 267-274, 1988.

BARRIGA, M. I. Microbial changes in shredded iceberg lettuce storage under controlled atmospheres. Journal of Food Science, Chicago, v. 56, n. 5, p. 15861588, 1991.

BOLLIN, E. M.; HUXSOLL, C. C. Effect of preparation procedures and storage parameters on quality retention of salad-cut lettuce. Journal of Food Science, Chicago, v. 56, n. 1 , p. $60-67,1991$. 
BRECHT, E. P. Use of controlled atmospheres to retard deterioration of produce. Food Technology, Chicago, v. 34, n. 3, p. 45-50, 1980.

FILGUEIRA, F. A. R. Novo manual de olericultura: agrotecnologia moderna na produção e comercialização de hortaliças. Viçosa: UFV, 2000. 402 p.

HAMZA, F. et al. Storage of minimally processed romaine lettuce under controlled atmosphere. Journal of Food Quality, Connecticut, v. 19, p. 177-188, 1996.

HEIMDAL, H. Biochemical changes and sensorial quality of shredded and MA-Packaged iceberg lettuce. Journal of Food Science, Chicago, v. 60, n. 6, p. 12651276, 1995.

JACKSEN. Behaviour of Listeria monocytogenes and Aeromonas ssp. on fresh-cut produce packaged under equilibrium modified atmosphere. Journal of Food Protection, Des Moines, v. 62, n. 10, p. 1128-1135, 1999.

KADER, A. A.; ZAGORY, D.; KERBEL, E. L. Modified atmospheres packaging of fruits and vegetables. Critical Reviews in Food Science and Nutrition, Chicago, v. 28, n. 1, p. 1-30, 1989.

KADER, A. A. Biochemical and physiological basis for effects of controlled and modified atmospheres on fruits and vegetables. Food Technology, Chicago, v. 40, n. 5, p. 99-104, 1986.

KADER, A. A.; LIPTON, J. W.; MORRIS, L. L. System for scoring quality of harvest lettuce. HortScience, California, v. 8, n. 5, p. 408-409, 1973.

LIPTON, W. J. Toward an explanation of disorders of vegetables induced by high $\mathrm{CO}_{2}$. Horticultural Rept., Michigan, v. 28, p. 137, 1977.

LOPEZ-GALVEZ,G. Quality changes in packaged salad products during storage. Z. Lebensm Unters Forsch A, [S.1.], v. 205, p. 64-72, 1997.

MATEOS; CANTWELL; KADER. Phenolic metabolism and fermentation of intact and cut lettuce exposed to $\mathrm{CO}_{2}$ : enriched atmospheres. Postharvest Biology and Technology, Chicago, v. 3, p. 225-233, 1993.
MEILGAARD, M.; CIVILlE, V. G.; CARR, B. T. Sensory evaluation techniques. 2. ed. Boston: CRP, 1991.

PANTASTICO, E. B. Postharvest physiology, handling and utilisation of tropical and subtropical fruits and vegetables. Connecticut: AVI, 1975.

ROLLE, R. S.; CHISM, G. W. Physiological consequences of minimally processed fruits and vegetables. Journal of Food Quality, Connecticut, v. 10, p. 157-177, 1987.

SARANTÓPOULOS, C. I. G. L. Embalagens com atmosfera modificada. Campinas: ITAL-CETEA, 1996.

SHEWFELT, R. L. Postharvest treatment for extending the shelf life of fruits and vegetables. Food Technology, Chicago, v. 40, n. 5, p. 70-85, 1986.

SHEWFELT, R. L. Quality of minimally processed fruits and vegetables. Journal of Food Quality, Connecticut, v. 10, p. 143-156, 1987.

SOUZA, J. P.; JONG, E. V.; GOULART, H. H. R. Aumente o tempo de conservação dos alimentos e obtenha maiores lucros. Porto Alegre: Imprensa Livre, 2001.

WILEY, R. Frutas y hortalizas mínimamente procesadas y refrigeradas. 20. ed. Tradução de José Fernández-Salguero Carretero. Zaragoza: Acribia, 1997.

YAMASHITA, F. Armazenagem frigorificada de mangas (Mangifera indica L. cv. Keitt) embaladas sob atmosfera modificada. 1995. $141 \mathrm{f}$. Tese (Doutorado em Engenharia de Alimentos) - Universidade Estadual de Campinas, Campinas, 1995.

YI WANG, C. Physiological and biochemical effects of controlled atmosphere on fruits and vegetables. In: CALDERON, M.; BARKAI-GALAN, R. Food preservation by modified atmosphere. [S.1.: s.n.], 1990. p. 197-223.

ZAGORY, D.; KADER, A. A. Modified atmosphere packaging of fresh products. Food Technology, Chicago, v. 42, n. 9, p. 70-77, 1988. 